\title{
APLICACIÓN DE FUNGICIDAS Y FERTILIZACIÓN NITROGENADA EN AVENA: EFECTOS SOBRE LA GENERACIÓN DE BIOMASA AÉREA Y RENDIMIENTO
}

\author{
DietZ, J. I. 1,2; SChieREnBECK, M.1,2; \\ MARTineZ, N. ${ }^{1} \&$ SimON, M. R..$^{1,3}$
}

\begin{abstract}
RESUMEN
El objetivo fue evaluar el efecto de la fertilización nitrogenada (N) y la aplicación de fungicidas sobre la severidad causada por enfermedades foliares, índice de área foliar, biomasa aérea y rendimiento en avena. Se condujo un ensayo en la Facultad de Ciencias Agrarias y Forestales (FCAyF), UNLP, con diseño experimental en parcela sub-sub-sub dividida y tres repeticiones, siendo la parcela principal: dos ambientes (2011-2014), la sub-parcela: los tratamientos con y sin fungicidas, la sub-sub-parcela: los tratamientos con 0 y $60 \mathrm{~kg} \mathrm{~N} \cdot \mathrm{ha}^{-1}$; y la sub-sub-sub-parcela: tres genotipos (Líneas 5 y 6 de la FCAyF, y Bonaerense INTA Calén). La aplicación de fungicida disminuyó la severidad y la fertilización $\mathrm{N}$ la aumentó en EC8.2, estadio en que la roya de la hoja incrementó sus valores. En dicho estadio, el fungicida ejerció un mejor control en el tratamiento sin N. Tanto la aplicación de fungicida como de $\mathrm{N}$ incrementaron el índice de área foliar, la biomasa aérea y el rendimiento. Las Líneas 5 y 6 presentaron un menor nivel de enfermedad, mayor biomasa y rendimiento que Calén.
\end{abstract}

Palabras clave: triazoles-estrobilurinas, nitrógeno, biomasa, rendimiento, Avena sativa.

\begin{abstract}
Fungicide applications and nitrogen fertilization in oat.

The present study was designed to determine the effect of nitrogen fertilization (N) and fungicide applications on the severity caused by foliar diseases and its effects on the leaf area index, biomass production and yield in oat. Field experiments were carried out in at the Facultad de Ciencias Agrarias y Forestales (FCAyF), UNLP, using a split-split-split-plot design with three replications. The main plots were two environments: 2014-2015, the subplots were the treatments: with and without fungicide, the sub-plots were the treatments with 0 and $60 \mathrm{~kg} \mathrm{~N} \cdot \mathrm{ha}^{-1}$. The sub-subplots were three oats genotypes (L5 and L6 from FCAyF and Bonaerense INTA Calén). Fungicide applications caused a reduction on the severity of the diseases and $\mathrm{N}$ tended to
\end{abstract}

1.- Cerealicultura. Facultad de Ciencias Agrarias y Forestales (UNLP). 60 y 119. C.C. 31. La Plata, Provincia de Buenos Aires.

2.- CONICET, CCT La Plata, Argentina. Email: juanidietz@hotmail.com

3.- CIC Provincia de Buenos Aires.

Manuscrito recibido el 28 de julio de 2016 y aceptado para su publicación el 11 de agosto de 2016. 


\section{J. I. Dietz et al.}

increase \% severity at growth stage (GS) 82, GS where leaf rust was preponderant. At this GS there was a better control produced by the fungicide without $\mathrm{N}$ applications. Both the fungicide and the $\mathrm{N}$ application increase leaf area index, biomass and grain yield. L5 and L6 genotypes showed lower severity values and higher biomass and yield production than INTA Calén.

Key words: triazols-strobilurins, nitrogen, biomass, yield, Avena sativa.

\section{INTRODUCCIÓN}

La avena (Avena sativa L.) es un cultivo de importancia debido a la diversidad de usos y tipos de productos que pueden obtenerse: grano, forraje verde, forraje conservado (heno y ensilaje), doble propósito (verde-grano) y uso del rastrojo. En Argentina se cultivan aproximadamente 1,3 millones de hectáreas, destinándose la mayor parte para uso forrajero. La provincia con mayor superficie de cultivo es Buenos Aires, seguida por La Pampa y Córdoba.

El manejo de adversidades es fundamental para obtener cultivos con altos rendimientos y calidad. Tanto la biomasa como la calidad de forraje y grano son afectadas por patógenos fúngicos, siendo las royas las enfermedades más ampliamente conocidas y destructivas de este cereal. Estas se presentan en casi todas las áreas del mundo en donde se cultiva avena, afectando a cualquier parte de la planta que se encuentre sobre la superficie del suelo, desde la etapa de plántula hasta el llenado de grano (17). Se destaca la «roya de la hoja» (Puccinia coronata f. sp. avenae P. Syd \& Syd) como la enfermedad fúngica más importante en el mundo. Las pérdidas provocadas por roya de la hoja pueden llegar al $32 \%$ de la materia seca y a un $26 \%$ en producción de grano (10).

Otro de los patógenos de importancia que afecta al cultivo es Pyrenophora ave- nae Ito et Kurib, anamorfo Drechslera avenae (Eidam) Sharif (causal de mancha de la hoja). Un aspecto fundamental para el manejo de las enfermedades foliares (principalmente roya de la hoja), es el uso de variedades resistentes; sin embargo, la mayoría de los cultivares de avena disponibles en nuestro país se comportan como moderadamente resistentes a susceptibles. Un método usual de control es la aplicación de fungicidas del grupo de los triazoles y estrobilurinas, que permiten controlar los patógenos, reduciendo las pérdidas de rendimiento.

El agregado de fertilizantes nitrogenados $(\mathrm{N})$ es otra de las prácticas difundidas en el cultivo. La fertilización $\mathrm{N}$ produce un rápido crecimiento y un gran aumento de producción de materia seca, y la respuesta varía básicamente de acuerdo a la fuente de $\mathrm{N}$ empleada, el momento de aplicación, la dosis y contenido de humedad y nitratos del suelo (4). Sin embargo, una mayor dotación de $\mathrm{N}$ puede favorecer el desarrollo de enfermedades biotróficas como las royas (15).

La información sobre el efecto conjunto que ejerce la fertilización $\mathrm{N}$ sobre las enfermedades foliares de la avena y la acción que ejercen los fungicidas en el control de las mismas es escasa. En un estudio realizado por Soovali et al. (2010), se demostró que aumentos en los niveles de fertilización $\mathrm{N}$, incrementaron la intensidad de Puccinia coronata y Pyrenophora avenae. Sin embargo, cuando distintas dosis de fertili- 